\title{
Reevaluación del lactante hospitalizado por diarrea aguda con deshidratación
}

\author{
Dr. José Luis Roessler B. ${ }^{1}$; Dra. Magdatena Araya Q. ${ }^{2}$; Dr. Julio Espinoza M. $^{2}$; \\ T.M. Isolda Pacheco M. ${ }^{2}$; Dra. Isabel Courard D. ${ }^{1}$; Dr. Oscar Brunser I. ${ }^{2}$
}

\section{Assessment of in-hospital management for children under 2 years of age with acute diarrhea}

The charts of 159 children under 2 year 3 of age, admitted for acute diarrhea to Hospital Félix Bulnes between May 1985-Aptil 1986, were retrospectively evaluated. Emphasis was given to the route employed to tehydrate them. Infants aged less than 6 months and those with birth weight under 3,000 required more frequent admissions (p $<0.0001$ ). Only $7.3 \%$ of patients under 3 montls of age were breastfed, in contrast to $80 \%$ of their non-hospitalized peers. On admission, Weight/Age was below $80 \%$ of the NCHS standard in $43.7 \%$ of patients: this proportion increased to $50 \%$ at the time of discharge. Only 4 children $(2.5 \%)$ were not dehydrated an admission, all had been admited by social reasons. About $90 \%$ of dehydrated patients received i.v, fluids. In $17 \%$ of adnit ted children, dehydration was mild with normal serum sodium and potasium levels: these patients stayed in hospital for $\bar{\mp} 16.9$ days, they lost $\mp 3.5 \%$ of their Weight/Age adequacy, and suffered 10 episodes of intercurrent infections while in hospital. This type of patients would benefit from an ORT program, which should reduce their needs for admission, risks of intercurrent diseases and costs of treatment.

(Key words: acute diatrhea, dehydration, in-hospital management, complications.)

La mortalidad infantil por diarrea aguda ha disminuido considerablemente en los últimos afios. Sin embargo, aún continúa representan. do la segunda causa de hospitalización y consul. ta en policlínicas periféricas en nuestro medio ${ }^{1 / 4}$. En la actualidad llama la atención que en nuestro pais los programas de hidratación oral para niños con diarrea aguda son escasos y, en general, obedecer a esfuetzos puntuales. Por este motivo hemos considerado de interés evaluar las características de los pacientes hospitalizados por diarrea aguda, de manera de obtener la información necesaria para fundamentar la revisión de algunas prácticas actuales y proponer acciones para el mejor tratamiento de estos pacientes, si es posible, evitando la hospitalización.

\section{MATERIAL Y METODOS}

El Hospital Félix Bulnes ofrece atención secundaria a una población que, entre 1985-1986, era de 27.101 nĭ̃os menorcs de dos años.

1. Servicin de Pedjatria del Hospital l'élix Bulnes.

2. Unidad de Gastroenterología, Instituto de Nurrjción y Tecnología de los Alimentos (INTA), Universidad de Chile.
Entre mayo de 1985 y abril de 1986 egresaron 1.370 niños del Servicio de Pediatría del Hospital Félix Bulnes. De éstos, $159(11,6 \%)$ correspondían a menores de 2 afos cuyo diagnóstico de ingreso era síndrome diarreico agudo $y$ deshidratación. Mediante un formulario precodificado se revisaron restrospectivamente las fichas clínicas de estos últimos, registrando en cada caso la fecha de nacimiento, pesos (a) nacer, al inicio de los síntomas y signos, en el ingreso al hospital, en el momento de hidratatse, al término de los sintomas y al alta), los diagnósticos de ingreso y egreso, la historia almentaria previa y durante la hospitalización; los agentes microbjológicos identificados durante el episodio, el tratamiento antibiótico adninistrado, los estudios de laborator jo efectuados, el tipo y grado de deshidratación, las técnicas utilizadas para la rehidratación y realimentación, la aparición de intolerancia por alimentos y de complicaciones. Los resultados se anatizacon en computador. Se utilizó un paquete de análisis estadístico ([BM-PC) para calcular promedios, desviaciones estandar, chi cuadrado y prueba de Fisher según se consideró necesario. La magnitud de la deshidratación se calificó con criterios clínicos ${ }^{5}$. Para clasificar las concentraciones sésicas de $\mathrm{Na}^{+}$y $\mathrm{K}^{+}$se consideraron como valores normales de $\mathrm{Na}^{+} 130$ a $149 \mathrm{mEq} / \mathrm{L}$ y de $\mathbf{K}^{+} 3,5$ a $5,5 \mathrm{mEq} / \mathrm{L}$. La intolerancia por disacáridos (lactosa) fue evaluada indirectamente con mediciones de $\mathrm{pH}$ y reacciones de Benedict para cuerpos reductores en las deposiciones $(\mathrm{pH}<5$ y reacción positiva de Benedict en deposiciones frescas obtenidas por sonda rectal). 


\section{RESULTADOS}

La distribución por edad de los menores de dos años hospitalizados por diarrea aguda y la de los niños del mismo grupo etario que reciben atención médica en el área que atiende el hospital es la de la tabla 1. En 7 pacientes no se encontró la información. Los mezores de 6 meses requirieron ser hospitalizados por diarrea con una frecuencia significativamente mayor ( $\mathrm{p}<0,0001)$. La edad promedio de hospitaliza. ción por está causa fue 5,2 meses, la mediana 4,4 meses y $51,5 \%$ de los niños eran varones. Comparando con el total de recién nacidos vivos en la maternidad del Hospital Félix Bulnes, durante el mismo período de tiempo, la proporción de niños con peso de nacimiento inferior a $3.000 \mathrm{~g}$ y a $2.500 \mathrm{~g}$ fue significativamente mayor entre los que requirieron hospitalización $(37,2$ vs. $18,0 \%$ y 14,5 vs. $7,0 \%, p<0,0001$, respectivamente). En 50 lactantes menores de tres meses en que se pudo establecer el tipo de alimentación, 7,3\% recibian pecho exclusivo, mientras que en los consultorios periféricos del área aproximadamente $80 \%$ de los lactantes de la misna edad eran amamantados. La distribución estacional de los ingresos fue: $27,35 \%$ en verano; $29,1 \%$ ell otoño, $10,7 \%$ en invierno, y $22,0 \%$ en primavera.

A] ingresar, $88,3 \%$ de los pacientes había sufrido diarrea por 7 dias o menos y 4,I $\%$ por 15 o más días. La información registrada en las fichas permitió calcular, solamente, la relación peso/edad de acuerdo a las tablas del National Center for Health Statistics (NCHS), en 135 niños, al ingreso (24 horas después de calificar al niño como hidratado) y 130 al alta. Aproxima. damente $70 \%$ de los pacientes evaluados estaban por debajo del $90 \%$ de adecuación al ingresar y 74\% de ellos al egresar (tabla 2).

Tabla 1

Distribución por edad de la población encargada al Hospital Félix Bulnes Bulnes y de 152/159 lactantes hospitalizados en el establecimiento por diarrea aguda. Período 1985-1986

\begin{tabular}{|c|c|c|c|c|}
\hline \multirow[t]{2}{*}{ Fdad } & \multicolumn{2}{|c|}{ Población a cargo } & \multicolumn{2}{|c|}{ Nitios hospitalizados } \\
\hline & No & $\%$ & No & $\%$ \\
\hline 6 meses & 6.890 & $(25,4)$ & 101 * & $(66,5)$ \\
\hline $6-11$ meses & 6.717 & $(24,8)$ & 42 & $(27,6)$ \\
\hline $12-14$ meses & 13.494 & $(49.8)$ & 9 & $(5,9)$ \\
\hline TOTAL & 27.101 & $(100,0)$ & 152 & $(100,0)$ \\
\hline
\end{tabular}

$\mathrm{p}<0,0001$.

Tabla 2

Relación peso/edad en niños hospitalizados por diarrea aguda al ingreso ( $x$ =35) (después de hidratados) $y$ al alta $(n=130)$ oon registro suficiente de información

\begin{tabular}{|c|c|c|c|c|}
\hline \multirow{2}{*}{$\begin{array}{l}\text { Peso/edad } \\
\text { \% del estándar } \\
\text { para la edad (NCHS) }\end{array}$} & \multicolumn{2}{|c|}{ Ingreso } & \multicolumn{2}{|c|}{ Alta } \\
\hline & No & $\%$ & No & $\%$ \\
\hline 110 & 3 & $(2,2)$ & 0 & $(0,0)$ \\
\hline $90-110$ & 37 & $(27,4)$ & 34 & $(26,0)$ \\
\hline $80-89$ & 36 & $(26,6)$ & 31 & $(24,0)$ \\
\hline $70-79$ & 25 & $(18,0)$ & 30 & $(23,0)$ \\
\hline 70 & 34 & $(25,8)$ & 35 & $(27,0)$ \\
\hline TOTAL & 135 & $(100,0)$ & 130 & $(100,0)$ \\
\hline
\end{tabular}


Sólo 4 niños $(2,5 \%)$ estaban hidratados al momento de ingresat: su hospitalización se había indicado por razones sociales. El $63,8 \%$, $27,7 \%$ y $5,8 \%$ de los pacientes presentaban deshidratación leve, moderada y severa, respectivamente. En 114 niños deshidratados se registró la concentración de electrólitos plasmá. ticos: $89(78 \%)$ tenian isonatremia, $9(8 \%)$ hipernatremia y $16(14 \%)$ hiponatremia. La concentración de $\mathrm{K}^{+}$fue normal en $53(46,5 \%)$, alta en $14(12,2 \%) y$ baja en $47(41,1 \%)$.

Con respecto a los estudios realizados al ingreso de los pacientes, en 122 mediciones de hemoglobina (efectuadas antes de hidratar al lactante), 65,5\% de los niños tenían menos de $11 \mathrm{~g} / \mathrm{dL}$. Los resultados de los coprocultivos, exámenes coproparasitológicos, estudios de rotavitus en deposiciones (por rotaforesis), urocultivos, hemocultivos y estudios en líquido cefalorraquídeo aparecen en las tablas 3 y 4 . Seis de los 9 hemocultivos positivos se obtuvieron entre los dias 13 y 47 de hospitalización, lo que sugiere que estas infecciones pudieron adquirirse dentro del hospital.

El recuento de leucocitos fecales se efectuó en 139 niños y resultó positivo en todos los pacientes con Salmonella y Shigella, en 11 de 16 casos con Escherichia coli (E. coli) $0111(68 \%)$, 18 de 31 casos con $E$. coli enteropatógena, se-

Tabla 3

Estudios etiológicos en deposiciones de lactantes hospitalizados por diarrea aguda

\begin{tabular}{|c|c|c|}
\hline & No & $\%$ \\
\hline \multicolumn{3}{|l|}{ Coprocultivos $\left(\mathrm{N}^{\circ}=122 / 159\right)$} \\
\hline $\begin{array}{l}\text { Escherichia coli enteropatógena } \\
\text { (Suero polivalente) }\end{array}$ & 37 & 30,3 \\
\hline Escherichia coll 0111 & 15 & 12,3 \\
\hline Shigella & 2 & 1,6 \\
\hline Salmonello & 2 & 1,6 \\
\hline Negativos & $6 \overline{6}$ & 54,1 \\
\hline TOTAL & 122 & 99,9 \\
\hline \multicolumn{3}{|l|}{ Parasitológico $\left(N^{\circ}=140 / 159\right)$} \\
\hline \multirow{2}{*}{$\begin{array}{l}\text { Giardia lamblia } \\
\text { Negativo }\end{array}$} & 1 & 0,7 \\
\hline & 139 & 97,3 \\
\hline TOTAL & 140 & 100,0 \\
\hline \multirow{2}{*}{$\begin{array}{l}\text { Rotaforesis } *\left(N^{\circ}=35 / 159\right) \\
\text { Negativos }\end{array}$} & 11 & 31,4 \\
\hline & 24 & 68,6 \\
\hline TOTAL & 35 & 100,0 \\
\hline
\end{tabular}

* Estudios efectuados entre noviembre y marzo. rotipos clásicos y en 6 de 11 niños, en que sólo se detectó rotavirus.

En 158 pacientes se pudo evaluar la vía de hidratación utilizada. En 99 casos $(62,6 \%)$ ésta se efectuó por yía endovenosa, en 12 pacientes $(7,6 \%$ ) por gastroclisis y mediante ambos procedimientos en 43 nifios $(27,2 \%)$. En 158 lactantes se buscaron cuerpos reductores en las deposiciones con resultados positivos en 42 casos $(26,6 \%)$. El diagnóstico de intolerancia se hizo, en promedio, a los 11,7 días de iniciada la diarrea (rango 1 a 42 días) y 6,5 djas después de haber ingresado al hospital (rango 1 a 20 días). La distribución etaria entre los lactantes con y $\sin$ cuerpos reductores fue comparable $(p=0,4)$; en cambio, la frecuencia de niños con cuerpos reductores fue significativamente mayor entre los niños desnutridos (peso/edad $\leqslant 80 \%$ del estándar NCHS para la edad, $p<0,005)$. No hubo correlación entre la detección de cuerpos reductores y los agentes etiológicos identificados.

La duración total del episodio pudo estimarse en 143 nifros y la de los síntomas en el hospital en 146 (tabla 5). En 32 pacientes $(22,4 \%$ ) la diarrea duró 15 días o más y en 8,9 la evolución intrahospitalaria excedió 14 días. En estos últimos no se demostraron diferencias significativas con los pacientes cuya evolución fue menor que 2 semanas en relación a la edad, estado de nu-

Tabla 4

Estudios etiológicos en orina, sangre y líquido cefalorraquídeo efectuados en lactantes hospitalizados por diarrea aguda

\begin{tabular}{|c|c|c|}
\hline & No & $\%$ \\
\hline \multicolumn{3}{|l|}{ Urocultivo $\left(\mathrm{N}^{\circ}=67\right)$} \\
\hline Escherichia coli & 3 & \\
\hline Proteus mirabilis & 2 & 3 \\
\hline \multirow[t]{2}{*}{ Negatjvo } & 62 & 92,5 \\
\hline & 67 & 1 \\
\hline
\end{tabular}

Hernocultivos $\left(\mathrm{N}^{\circ}=35\right)$

Escherichia coli

Klebsiello

Stephilococcus aureus

Doagulasa positivo

Enterobacfer cloacae

Pseudomonas aeruginoso

Negativos

\begin{tabular}{rrr}
4 & 11,4 \\
1 & 2,9 \\
& 1 & 2,9 \\
1 & 2,9 \\
2 & 5,7 \\
& 26 & 74,2 \\
\cline { 2 - 3 } TOTAL & 35 & 100,0
\end{tabular}

Líquido cefalorraquideo $\left(\mathrm{N}^{0}=6\right)$

Negativas

$6 \quad 100,0$ 
Tabla 5

Duración total e intrahospitalaria del episodio de diarrea aguda en lactantes con sindrome diarreico agudo con deshidratación (se incluyen sólo casos con información completa)

\begin{tabular}{|c|c|c|c|c|}
\hline \multirow[t]{2}{*}{ Tiempo (dias) } & \multicolumn{2}{|c|}{ Duración total del episodio diarreico } & \multicolumn{2}{|c|}{ Duración de los síntomas y signos desde el ingreso } \\
\hline & No & $\%$ & No & $\%$ \\
\hline $\begin{array}{r}7 \\
8-14 \\
15-21 \\
22\end{array}$ & $\begin{array}{l}52 \\
59 \\
20 \\
12\end{array}$ & $\begin{array}{r}36,3 \\
41,3 \\
14,0 \\
8,4\end{array}$ & $\begin{array}{r}106 \\
27 \\
5 \\
8\end{array}$ & $\begin{array}{r}72,6 \\
18,5 \\
3,4 \\
5,5\end{array}$ \\
\hline TOTAL & 143 & 100,0 & 146 & 100,0 \\
\hline
\end{tabular}

trición, peso de nacimiento, detección de leuco. citos fecales, estación del año en que se produjo su hospitalización, ni grado o tipo de alteración hidroelectrolítica al ingreso. Sin embargo, se observó una alta incidencia de cuerpos reductores en sus deposiciones: $77,0 \%$ versus $20,8 \%$ en los pacientes cuyo episodio duró menos de 2 semanas. Por otro lado, 4 de 9 septicemias detectadas ocurrieron en los pacientes con evolución prolongada. Los dos niños que fallecie. ron $(1,25 \%)$ sufrieron diarrea prolongada, desmutrición severa y afección genética (S. de Down en un caso y no precisada en otro).

Veintisiete niños, $17 \%$ de los ingresos, tenían deshidratación leve, isonatremia y normokalemia. Su evolución se caracterizó por un promedio de 16,9 dias de hospitalización (mediana 8 , rango 3 a 68 días); 1,5 días de hidratación y pérdidas de 3,5\% en la proporción de adecuación al estándar para la relación peso/edad durante la hospitalización. En el momento del alta se habían registrado 10 episodios de infecciones intrahospitalarias en $6(22,2 \%)$ de estos niños: 5 episodios nuevos de diarrea, 3 de varicela, 1 septicemia estafilocócica y 1 infección respiratoria alta. Por otro lado, durante la evolución de estos 27 niños, en cuatro se detectaron cuerpos reductores en las deposiciones, uno sufrió bronconeumonia, en dos se demostró infección urinaria y en uno el episodio de diarrea se hizo refractario al tratamiento.

\section{DISCUSION}

Este estudio consistió en la revisión retrospec. tiva de las fichas da niños hospitalizados por diarrea aguda, por lo que no fue posible analizar todas las variables incluidas en el instrumento precodificado creado para la evaluación. Los lactantes menores de 6 meses, de bajo peso al nacer y que han tenido menos de 3 meses de lactancia materna, tuvieron mayor riesgo de requerir hospitalización por diarrea aguda. Estos hallazgos coinciden con descripciones hechas en pacientes hospitalizados que desarrollan diarrea prolongada $^{6,7}$.

El análisis de la relación peso/edad demostró que los lactantes que ingresaron por diarrea agu. da presentaron una alta proporción de desnutrición, francamente mayor que la tasa observada en la población general. Por otro lado, durante la hospitalización este parámetro tendió a deterio. rarse. Estudios anteriores efectuados en Santiago por otros autores han demostrado resultados semejantes ${ }^{8-10}$. Es probable que las variaciones del estado de la nutrición tengan relación con la modalidad de hidratación y realimentación que se utilice durante el tratamiento del episodio de diarrea; sin embargo, como los datos de este estudio fueron obtenidos retrospectivamente, no permitieron analizar este aspecto en detalle. Otro hallazgo que sugiere que el estado de la nutrición de los pacientes es deficiente al ingreso es la alta proporción de niños con anemia. La frecuencia de deposiciones con $\mathrm{pH}$ ácido y reacción de Benedict positiva fue alta en el grupo evaluado $y$ significativamente mayor en los desnutridos.

El estudio etiológico fue incompleto en la mayoria de los casos. De las bacterias enteropatógenas no se buscó Campylobacter, que constjtuye uno de los agentes bacterianos más frecuentes asociados a diarrea ${ }^{1 \mathrm{~b}}{ }^{12}$. Tampoco se estudió la capacidad enteroinvasora y toxigéni. ca de las $E$. coli y el estudio de los serotipos clásicos incluyó sólo evaluación con sueros polivalentes en la mayoría de los pacientes. Los 
estudios coproparasitarios mostraron un porcentaje bajo de casos positivos. Esto coincide con otros trabajos nacionales y podría tener relación con la edad de los sujetos estudia. $\operatorname{dos}^{13}, 14$. La presencia de leucocitos fecales no guardó relación con el aislamiento de ningún enteropatógeno en especial.

Sólo 5,8\% de los niños ingresados mostraba al ingreso signos clínicos que suginietan contraindicaciones para recibir hidratación oral, ya que su. frían deshidratación grave y signos de choque ${ }^{15,16}$. Sin embargo, aproximadamente $90 \%$ de los pacientes fueron rehidratados por vía endovenosa. Por otro lado, 27 nifios (17\%) presentaban al ingreso deshidratación leve, nomonatrémica y normokalémica. Probablemente estos nifros habrían sido tratados ambulatoriamente si se hubiera contado con un programa adecuado de rehidratación oral. Si se toma en cuenta la duración de las hospitalizaciones, las enfermedades intercurrentes y el deterioro del estado de nutrición que sufrieron, resultan evidentes los beneficios que brindaría un programa que diera oportunidades de evitar la hospitalización de estos pacientes.

\section{RESUMEN}

Se evaluaron restrospectivamente, con énfasis en el manejo de su deshidratación, las caracteristicas de los 159 niños menores de 2 años hospitalizados por diarrea aguda entre mayo 1985 y abril 1986 en el Hospital Félix Bulnes. Los menores de 6 meses o con peso de nacjmiento inferior a $3.000 \mathrm{~g}$ requirieron hospitaljzarse con mayor frecuencia $(\mathrm{p}<0,0001)$. Sólo 7,3\% de los nifros menores de 3 meses recibían pecho exclusivo en comparación con $80 \%$ de sus pares no hospitalizados. En el ingreso, 43,7 por ciento de los niños tenían la relación pesol edad menor que $80 \%$ del estándar NCHS, 10 que aumentó a $50 \%$ al alta. Sólo 4 niffos $(2,5 \%)$ estaban hidratados al ingreso, el que se debió a razones sociales. De los deshidratados, alrededor de $90 \%$ recibieron hidratación parenteral. En 17\% de los niños habia, al ingresar, deshidratación leve, isonatrémica y normokalémica. Estos pacientes estuvieron hospitalizados en promedio 16,9 días, perdieron $\mp 3,5 \%$ de su adecuación peso/edad durante la hospitalización y presentaron $\mp 10$ episodios de infección intrahospitala. ria. Este tipo de paciente se beneficiaría con un programa de hidratación oral, que le brindaría la oportunidad de evitar la hospitalización.

\section{AGRADECIMIENTOS}

Los autores agradecen a la sefíorita Gloria Icaza y al señor Gabriel González por su ayuda en el análisis estadístico de los datos.

\section{REFERENCIAS}

I. Meneghello J., Rosselot J., Aguitó C., Monckeberg $F$, Undurroga $O$., Ferreiro $M$.: Infantile diarthea and dehydration: ambulatory treatment in a hydration center. Adv Pediatr 1960; 11: 183-1208.

2. Ministerio de Economí, Fomento y Reconstruccion, Instituto Nacional de Estadísticas: Demografía 1982, Chile. $1982 ; 63-92$.

3. Arayz M. Brunser O.: Country Report: Chile. Basic demographic information management and ptograms related to diarrheal disease in infantile population. International Nutritional Planners Forum. West Dean. Lnited Kingdom 1985; 1-27.

4. Biuocchi N., Aroyo $M_{\text {. }}$ Espinoza $J$, Brunser $O$. : Prevalencia de diarrea prolongada a nivel de un centro de saiud primario en Santiago. XXIII Reunión Anual de la Sociedad Latinoamericana de Investigación Pediátrica (SLAIP). Neuquén, Argentina, Abstract No 44, 1985.

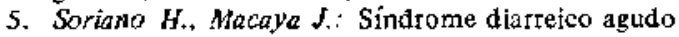
con deshidratación. En Meneghello J., Fanta E., Macaya J., Soriano H., Eds. Pediatría 3ra. Ed. Santiago, Chile. Mediterráneo 1985; 992-1003.

6. Rossi T.M. Lebenthal $E$.: Intractable diarhes of infancy. Fn: Lebenthal E. Texbook of gastroenterology and nutrition in infancy. New York, Raven Press 1981; 987-1001.

7. Barrera $F$, Curotto D., Avendaño P., Espinoza $A$., komero $G_{*}$, Escohor $S$ : Diarrea refractaria. Rev Chil Pediatr $1984 ; 55: 316-320$.

8. Anuario 1983: Defunciones y causas de muerte, Ministerio de Salud, Departamento de Control y Evaluación. Santiago, Chile, Imprenta del Ministerio de Salud $1984 ; 1-162$.

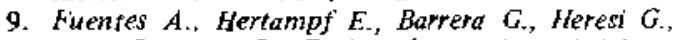
Jarpa S. Uauy $R$.: Evaluación nutricional del paciente pediátrico hospitalizado. Rey Chil Pediatr $1981 ; 52: 387-395$.

10. Gattas V., Fuentes A., Jarpa S., Uauy R.: Situación alimentaria de pacientes pediátricos hospitalizados. Rev Chil Pediatr 1981; 52: 397-404.

11. Duffat $G$., Lagos $R$., (jarcia $J$,, Maldonado A.: Campylobacter fetus subespecie yeyuni en lactantes con síndrome diarreico agudo. Rcv Chil Pediatr 1982; 53 : 205-212.

12. Figueroa $G$., Espinozo $J$. , Brunser O., Araya $\boldsymbol{M}$.: Campylobacter: cuatro años de experiencia en Chile. X Jornadas Nacionales de Pediatria, Viña del Mar, 1983. 
13. Zacarias J., Spencer E., Prado V., Herskovic P., Cohen $J .$, Tagle $S$., Cienfiuegos $G$., Araya $M$ : Recuperación fecal de rotavirus y otsos enteropatógenos en lactantes menores de 1 año con diarrea azuda. I. Verano. II. Invierno. Rev Chil Pediatr 1982; 53: $111-116$.

14. Figueroa G., Araya M., Ibánez S., Clerc N., Brtnser $O$. .: linteropathogens associated with acute diarrhea in hospitalized infants. J Pediatr Gastroentetol Nutr $1986 ; 5: 226-231$.

15. Klish $\boldsymbol{W}$.: Use of oral fluid in treatment on diarrhea. Pediatr Rev 1985: 7: 27-30.

16. Pizarro D., Posada G., Matta L.: Treatment of 242 neonates with dehydrating diarrhea with an oral glucose-electrolyte solution. J Pediatr 1983; 102: 153-156. 